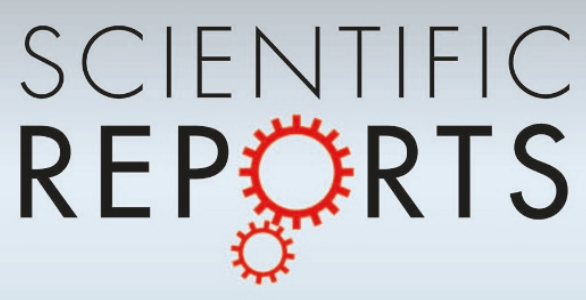

OPEN

SUBJECT AREAS:

APPLIED PHYSICS

MECHANICAL AND STRUCTURAL PROPERTIES AND DEVICES

SENSORS AND BIOSENSORS

SENSORS

Received

22 August 2012

Accepted

12 October 2012

Published

16 November 2012

Correspondence and requests for materials should be addressed to

H.W.Z.

(hongweizhu@ tsinghua.edu.cn) or Q.S.Z. (zhengqs@ tsinghua.edu.cn)

\footnotetext{
* These authors contributed equally to
} this work.

\section{Stretchable and highly sensitive graphene-on-polymer strain sensors}

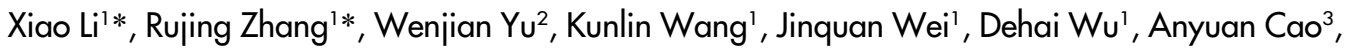 \\ Zhihong Li', Yao Cheng ${ }^{5}$, Quanshui Zheng ${ }^{5}$, Rodney S. Ruoff' \& Hongwei Zhu ${ }^{1,5}$
}

\begin{abstract}
'Department of Mechanical Engineering, Key Laboratory for Advanced Manufacturing by Materials Processing Technology, Tsinghua University, Beijing 100084, China, ${ }^{2}$ Department of Computer Science and Technology, Tsinghua University, Beijing 100084, China, ${ }^{3}$ Department of Materials Science and Engineering, College of Engineering, Peking University, Beijing 100871, China, ${ }^{4}$ National Key Laboratory of Science and Technology on Micro/Nano Fabrication, Institute of Microelectronics, Peking University, Beijing 100871, China, ${ }^{5}$ Center for Nano and Micro Mechanics (CNMM), Tsinghua University, Beijing 100084, China, ${ }^{6}$ Department of Mechanical Engineering and the Materials Science and Engineering Program, University of Texas at Austin, Austin, TX 78712, USA.
\end{abstract}

The use of nanomaterials for strain sensors has attracted attention due to their unique electromechanical properties. However, nanomaterials have yet to overcome many technological obstacles and thus are not yet the preferred material for strain sensors. In this work, we investigated graphene woven fabrics (GWFs) for strain sensing. Different than graphene films, GWFs undergo significant changes in their polycrystalline structures along with high-density crack formation and propagation mechanically deformed. The electrical resistance of GWFs increases exponentially with tensile strain with gauge factors of $\sim 10^{3}$ under $2 \sim 6 \%$ strains and $\sim 10^{6}$ under higher strains that are the highest thus far reported, due to its woven mesh configuration and fracture behavior, making it an ideal structure for sensing tensile deformation by changes in strain. The main mechanism is investigated, resulting in a theoretical model that predicts very well the observed behavior.

train sensors measure local deformations and are used mainly for damage detection, characterization of structures and fatigue studies of materials. Traditional sensors (metal and semiconductor strain gauges) have high sensitivities and can be low cost. But they have drawbacks. Most are fixed directional sensors and strain can only be measured in a specific direction; they have low resolution at the nanoscale and cannot be embedded in structural materials. Sensors based on nanomaterials (e.g. nanoparticles ${ }^{1}$, nanotubes ${ }^{2}$, nanowires ${ }^{3-5}$, thin films ${ }^{6-8}$ ) and their assemblages have been attracting interest recently due to their strain sensing characteristics. For example, strain sensors comprised of carbon nanotubes (CNTs) $)^{6-14}$, zinc oxide nanowires ${ }^{4,5}$, or graphene $^{15-21}$ serve as good alternatives for developing new sensors because of their outstanding properties. For graphene-based sensors, the principal vibrational frequencies ${ }^{15}$ and electrical conductance ${ }^{16}$ of graphene strongly depend on its topological structure which can be modulated by applying uniaxial strain, making it useful for high sensitivity tensile strain sensing. Moreover, nanomaterials can be embedded into structural materials and operate as both multidirectional and multifunctional sensors with high strain resolution at the nanoscale. The electromechanical properties of these strain sensors exhibit excellent characteristics compared to the traditional sensors due to a combination of high elastic moduli and outstanding electrical properties.

Our previous study showed that graphene woven fabric (GWF) might be an ideal component for strain sensors due to its special mesh structure composed of woven graphene microribbons (GMRs) ${ }^{22}$. In this work, GWFs have been embedded into polymers or used as patches on the surface of structural materials (like normal strain gauges). GWFs have a stable and predictable resistance response as a function of strain. They can measure very high strain (up to 10\%) and are well suited to highly stressed hybrid configurations, with significant resistance changes of 10 times at $2 \%$ and 10,000 times at $8 \%$. The main mechanism is investigated, resulting in a theoretical model that predicts very well the observed behavior.

\section{Results}

Tensile test of GWF/PDMS films. As shown in Figure 1a, a GWF thin film was coated onto or embedded in a poly(dimethylsiloxane) (PDMS) matrix. Then the composite was subjected to external loading. Figure $1 \mathrm{~b}$ shows a wired and a bent sample. The main feature of interest for tensile tests on the GWF-on-PDMS device is the 



Figure 1 | GWF-on-PDMS structure for tensile test. (a) Schematic of the GWF-on-PDMS structure. (b) Macroscopic optical image of a wired sample. (c) A series of optical images showing the formation of crack and their evolution in GWF under different strain, and corresponding schematics. (d) Optical images of the GWF under large strains (20\% and 50\%).

formation of high-density cracks that were initiated at weak points under small strains. The crack length and crack density gradually increased with increasing strain (Figure 1c). At large strains, cracks are clearly seen in low magnification optical images and are predominantly perpendicular to the tensile direction, and are uniformly distributed in the stretched GWF. Finally, the cracks continue to propagate leading to the fracture of the GMRs (Figure 1d). After the removal of the external force, the cracks disappear and the fractured GWF recovers to the initial state. The electrical resistance was monitored in real time. When a GWF was stretched within the limits of the underlying substrate's elasticity, its resistance increased and significant changes were observed during deformation (loading and unloading) that makes GWF-on-PDMS films applicable for strain sensing applications.

Figure $2 \mathrm{a}$ characterizes the resistive response of the composite sensor to the external loading; the electrical resistance monotonously increases with the applied strain, behaving like a variable resistor, but the slope of the relative resistance $\left(\Delta R / R_{0}\right)$ curve has three stages, all in approximately exponential fashions. At the first stage, the relative electrical resistance increases significantly and shows a highly nonlinear relation with strain up to approximately $1 \%$ strain, caused by the initialization and fast propagation of crack in the GMRs in the GWF network. At higher strains, the relative change in resistance under strain still rises exponentially due to the development of cracks and because of the dimensional change of the GWF networks. Moreover, the slope of the resistance-strain curve also increases exponentially with strain, suggesting an irreversible resistance transition, associated with extensive concentration of stresses in interphases and continuous fracture of GMRs. $\Delta R / R_{0}$ values are $\sim 1$ at $0.5 \%, 5 \sim 10$ at $2 \%$ and $10^{3} \sim 10^{4}$ at $8 \%$ depending on the crystallinity of GMRs. At the third stage, owing to the growth of cracks in the GWF, the neighboring GMRs are completely disconnected and the resistance jumps to "infinity" (out of measurable range) at strains of $15 \sim 20 \%$.

As shown in Figure 2b, it can be seen that under the saw-tooth wave strain, the current switched rapidly at every turning point, and the current remained nearly constant at the same value of strain. The sensor maintained these superb response and recovery properties even under high strains of $<10 \%$, suggesting applications such as for precision measurements. Tested with different frequencies of strain $(0.02 \sim 1 \mathrm{~Hz})$, the GWF-on-PDMS sensor also shows almost no frequency dependence of the current change at the applied strain (Figure 2c). Figure $\mathrm{S} 1$ shows the recovered resistance of the GWF-onPDMS sensor upon cycling under different strains, demonstrating its excellent stability and robustness after 100 -cycle tests.

The woven mesh structure of GWF is highly sensitive to deformation. For comparison, Figure $2 \mathrm{~d}$ gives the current and relative resistance (inset) as a function of strain for a graphene film. Different than the GWF, the film resistance increases almost linearly with strain, with moderate changes of 2 times at $2 \%$ and only 7 times at $8 \%$.

Gauge factors. The slope of the resistance-strain curve of GWF (see the inset of Figure 2a), reflects the gauge factor of a sensor, defined as $(\mathrm{d} R / R) /(\mathrm{d} L / L)$, where $R$ and $L$ are the resistance and length of the sensor, respectively. As shown in Figure 3, the gauge factors of a GWF sensor are calculated to be $\sim 10^{3}$ under $2 \sim 6 \%$ strains and $10^{6}$ under higher strains $(>7 \%)$. These values are to our knowledge the highest thus far reported, higher than the gauge factors $(0.06$ $\sim 0.82$ ) for $\mathrm{CNT} /$ polymer composites ${ }^{12}, 1 \sim 5$ for conventional metal gauges $^{23}, \sim 20$ for carbon black (50wt\%)/polymer composites ${ }^{24}, \sim 100$ for nanowire/polystyrene hybrid films ${ }^{5}, \sim 200$ for a doped Si strain sensor, $\sim 1000$ for a nanotube based sensor ${ }^{25}, 1250$ for a single nanowire based sensor ${ }^{4}$.

\section{Discussion}

To understand the underlying mechanism of the GWF 'stretchability' and explain the electromechanical response of GWFs during tensile tests, the structural changes occurring in GWF under different levels of strain were examined. We first explain why the abovementioned cracking phenomenon may happen, and then show that the cracking propagation would be main mechanism of the observed high sensitivity of the GWF-on-PDMS sensors to strain.

On initial stretching, irreversible fracturing throughout the GWF created cracks; with further strain, the crack density and also their widths increased, explaining the observed exponential increase in resistance. Uniformity of the GMRs was vital for homogeneous 

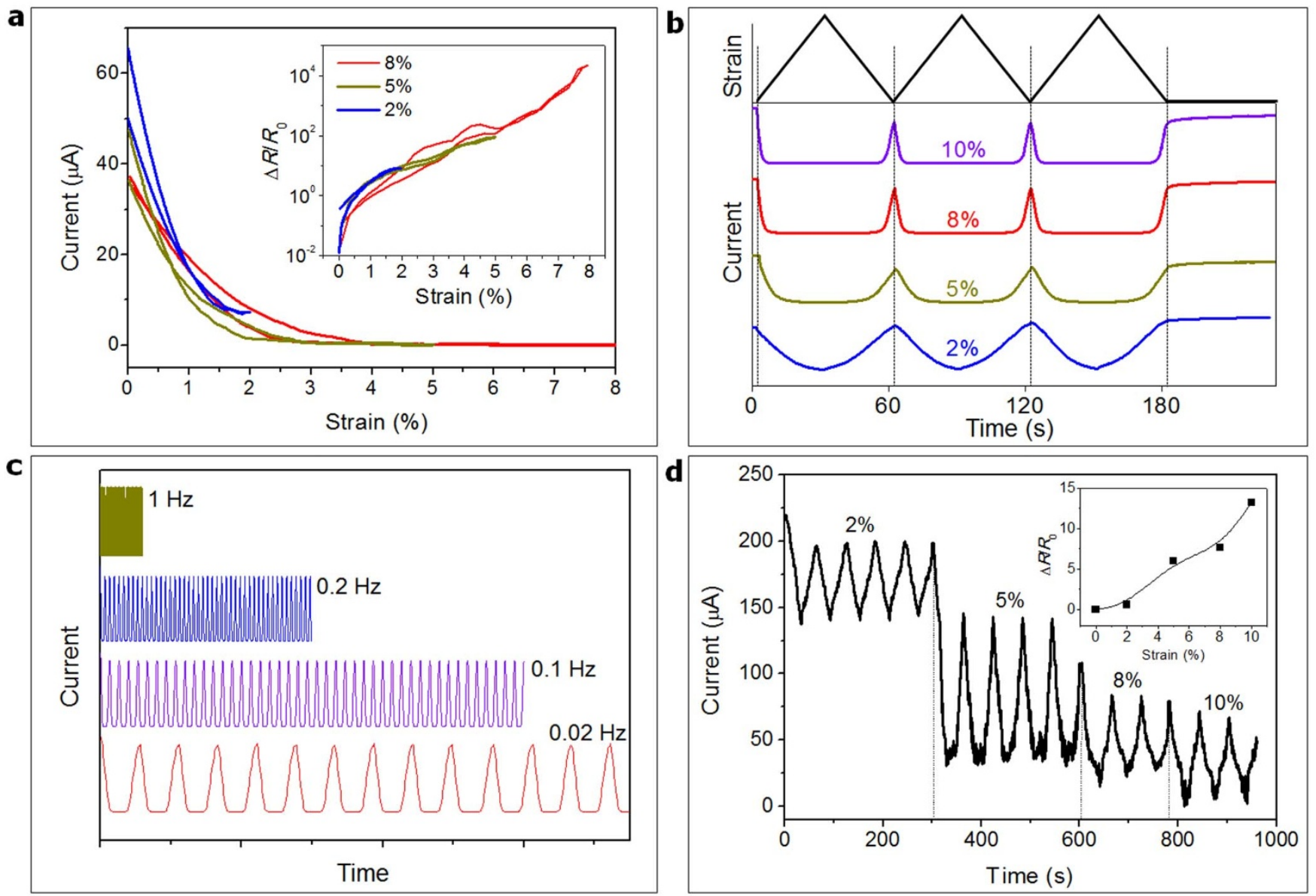

Figure 2 Electromechanical behavior of the graphene-on-PDMS strain sensors. (a) The changes in current and resistance (inset) under different strain. (b) Current response at different static strain. (c) Current response at different frequency under 5\% strain. (d) Current and relative changes in resistance (inset) of graphene film. All samples were tested under a bias of $1 \mathrm{~V}$.

fracturing throughout the GWF. In addition, the GWF showed buckling parallel to the strain axis, because its deformation followed the same positive Poisson's ratio as the PDMS substrate. This highlighted the importance of the woven structure of the GWF in allowing a super exponential resistive response.

Our chemical vapor deposition (CVD) grown graphene has an overlapped polycrystalline nature, as illustrated in Figure 4a. Asgrown graphene is composed of single crystalline graphene sheets with an overlapped region between any two adjacent sheets and overlapping width ranging from about 50 to $200 \mathrm{~nm}$. The measured

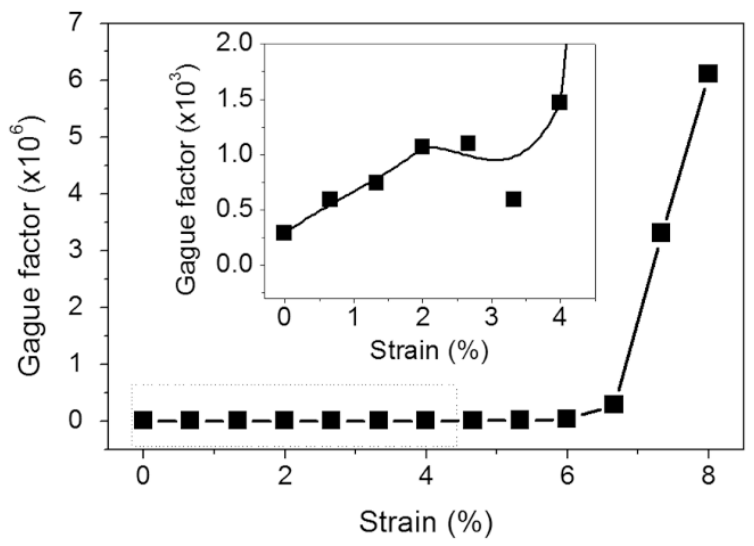

Figure $3 \mid$ Gauge factors of the GWF-on-PDMS strain sensor. sheet size distribution is shown in Figure S2, with mean size about $5 \mu \mathrm{m}$. During a large stretching, the PDMS will response to almost the same stretching while the graphene sheets adhered to the PDMS will keep almost inextensible since its Young's modulus is more than $10^{6}$ times of that of a PDMS and the strength against relatively sliding between graphene sheets and the underlying PDMS is fairly low. Accordingly, if we ignore the stretching of the graphene sheet, then the overlapping width between two adjacent graphene sheets of sizes $L_{1}$ and $L_{2}$ will shrink from the initial width, $w_{0}$ to $w_{0}-L \varepsilon$, where $L=$ $\left(L_{1}+L_{2}\right) / 2$. The critical strain that is required to yield separation or "crack" initiative is thus equal to $\varepsilon_{\mathrm{cr}}=w_{0} / L$, as depicted in the bottom panel of Figure $4 \mathrm{a}$. The crack information (density, length) agrees well with the microscopic observation of crack formation within GWF under different strains.

As illustrated in Figures $4 \mathrm{~b}$ and 4c, the GWF forms an electrical network with a variable resistor assembled to each current pathway. A partial crack perpendicular to a pathway will lead to an increase of the resistance while an across crack will result in breaking off the pathway. To understand the cracking effect, a program was developed to solve the equivalent resistor network and output the resistance (see Experimental for details). With the microscopic observation of crack formation within GWF under different strains, the relationship between the density of cracks and the strain was first established. Figure $4 \mathrm{c}$ shows a schematic model of the current pathway within the GWF. It was found that cracks are uniformly distributed in the stretched GWF, revealing the intrinsic polycrystalline feature of CVD-grown graphene. After applying the statistical crack information (density, length) derived from Figure 4a and Figure S2 to 
a

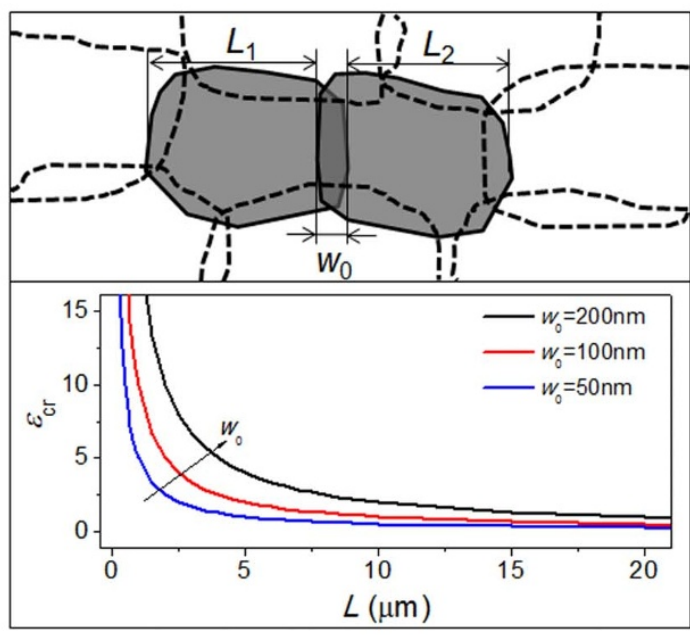

C

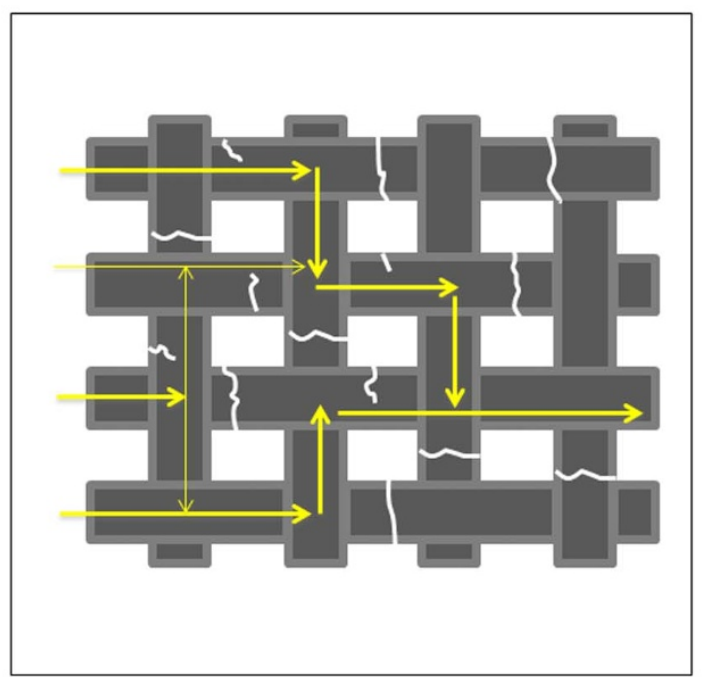

b

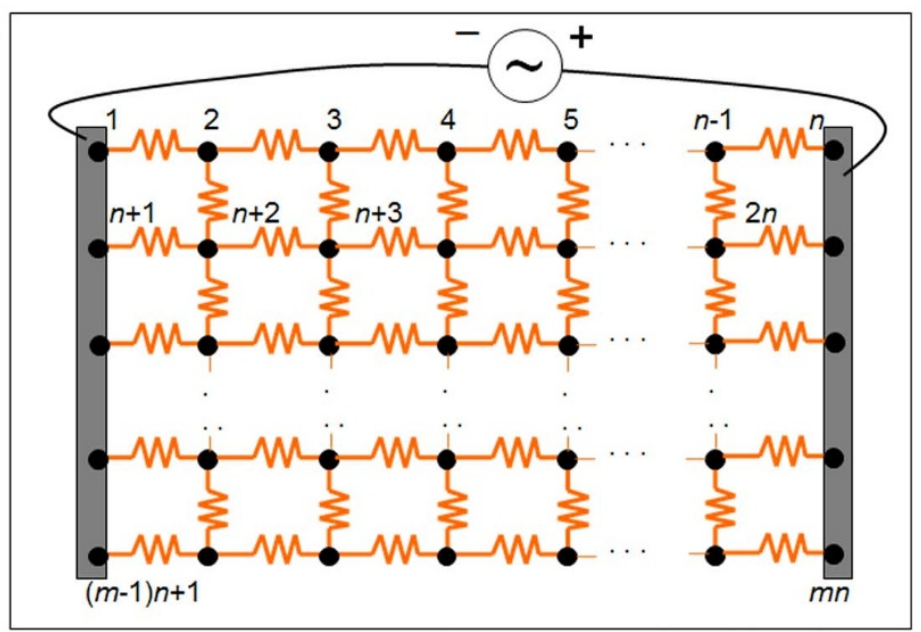

d

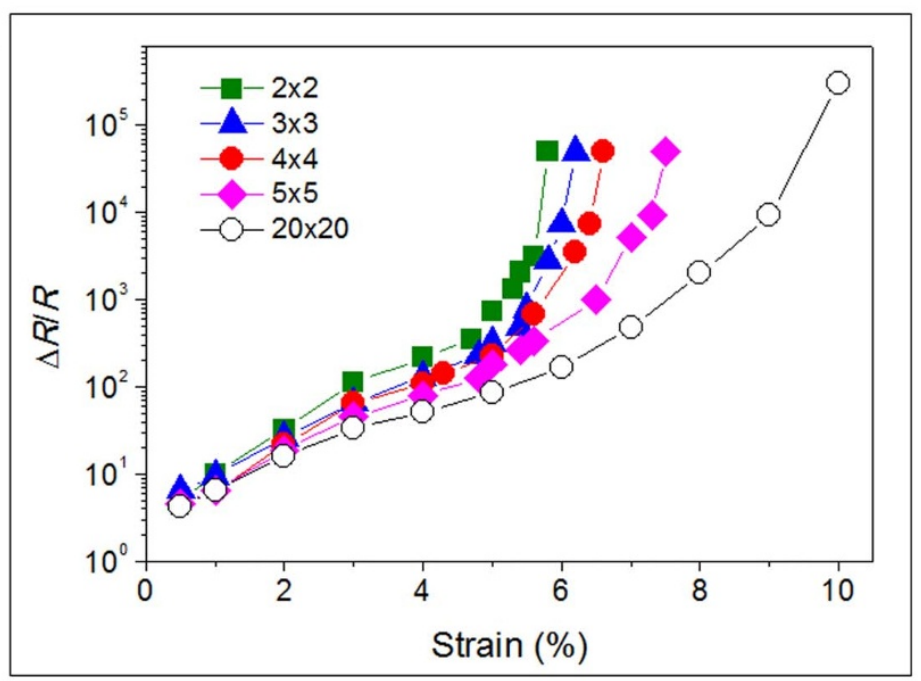

Figure $4 \mid$ Fracture model of GWF. (a) Schematic structure of polycrystalline graphene (top) and the critical strain versus graphene sheet size plot (bottom). (b) The equivalent circuit model for estimating the resistance of GWF's with specified cracked GMRs. (c) Current pathway through a fractured GWF. (d) Calculated resistance changes of GWFs with different configurations.

the circuit model, the resistance of GWF under a specific strain was calculated. Figure $4 \mathrm{~d}$ shows the resistance changes versus strain for GWF samples of different configurations. Similar to previously reported multi-fiber embedded composites with improved sensitivity ${ }^{11}$, the resistance of the GWF-on-PDMS strain sensor increases sharply with the many segments of GMRs gradually broken one by one or alternately, which acted as multi variable resistors connected in matrix. $\Delta R / R_{0}$ shows an exponential increase with increasing strain, in good agreement with the experimental results. With increasing number of GMRs embedded in the parallel and woven structures, the stretched samples display enhanced ability to sustain high strains. The simulation well explained the mechanism of the remarkable resistance change with respect to strain in GWFs, and the high strain sensing could be attributed to the gradual breaking of GMRs upon stretching.

Although the calculated resistance increases exponentially with strain, it is about an order of magnitude lower than experimental values for the GWF composed of $20 \times 20$ GMRs. The difference grows with the strain and the number of GMRs. Especially, the experimental results show a rapid increase in $\Delta R / R_{0}$ at low strains (Fig. 2a). There are two reasons for this. First, the model employed here only considers cracks in the GMRs in the weft direction, which is parallel to the load. However, a great many cracks also grow in the GMRs perpendicular to the load, due to the compression during the tensile process. The contact resistance could be relatively large, having a significant impact on the total resistance. Since the GMRs form a multi-joint network, cracks parallel to the stress may emerge on GMRs in the warp direction under large strain as well. Second, the observed distribution of cracks cannot reflect all aspects of crack growth and propagation, since the resolution of optical and even the electron microscope is limited. Meanwhile, GMRs with lots of initial defects will break completely under low strain, resulting in a significant increase in resistance. The general behavior is demonstrated here, and the mechanism of the exponential change of resistance to strain is well rationalized.

The resistance response of GWF is tunable to some extent. The crack formation is affected not only by the original graphene quality, but also by the GWF fabrication process, resulting in variations in sensing characteristics when their layouts (e.g. area, grid density) are different or stretched along different direction. For example, when a prestretched GWF is tested or a GWF is stretched along the XY direction, the exponential change of resistance to strain becomes weaker $^{22}$. The mechanism of stretchability along the XY direction is analogous to the structural deformation of open-mesh geometries used to wrap two-dimensional objects. When the frame is stretched, open rectangular holes deform to allow stretching, while the strips act as bending units. This process is downsized with a relative change of resistance of $\sim 5$ at $2 \%$ strain and $\sim 100$ at $10 \%$ strain for the GWF 

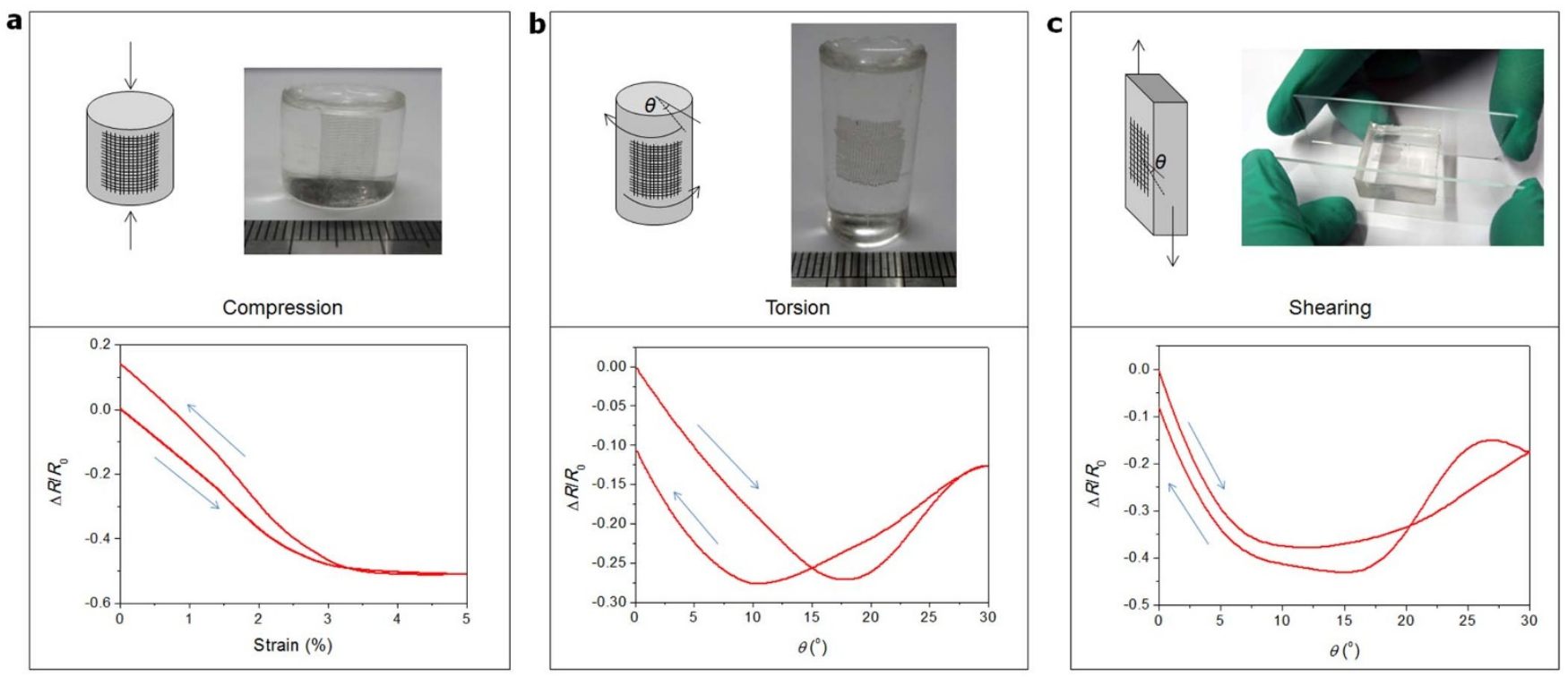

Figure $5 \mid$ Universal strain sensing. (a) Compression. (b) Torsion. (c) Shearing. Top panels: schematics and photographs, bottom panels: corresponding changes in resistance under different deformation. The sensing behavior depends on the dimensions of PDMS and GWF. All GWF samples are $\sim 1 \times 1 \mathrm{~cm}^{2}$.

(Figure S3). Several tensile tests were conducted on different types of GWF sensors (Fig. S4). The experimental result reveals that the area and grid density mainly influence the stability of the sensor, and the tensile direction mainly influence the sensitivity of the sensor.

To further reveal the potential of GWFs for use in tensile strain sensors, a variety of strain sensing experiments using GWF-onPDMS sensors, such as compression, torsion, and shearing, were conducted and the results are shown in Figure 5. As a GWF consists of discontinuous graphene sheets, the GWF-on-PDMS strain sensor can be considered a defective graphene mesh reinforced with a solid surface. Due to it polycrystalline structure, some micro-cracks were generated in the GWF during wet-chemical based transfer and subsequent drying. When the GWF was compressed such that it buckled, these cracks were recovered, resulting in a decrease in resistance (Figure $5 \mathrm{a}$ ). Broken sheets could remain very close to each other because they stuck to the PDMS substrate, enabling them to rejoin when the external load is released. Similarly, as for torsion and shearing, the cracks first merged then regrew with further deformation. Therefore, as shown in Figure $5 b$ and $5 c$, the resistances drop at early stages of loading then increase. It is worth noting that the relative resistances for these three deformations are negligible $(\Delta R /$ $\left.R_{0}<0.5\right)$ compared to tensile deformation, suggesting its sensitivity as a tensile strain sensor that is less influenced by other deformations.

In summary, GWF-on-polymer composite films, fabricated by directly coating GWFs on selected polymers, hold great promise as strain sensors that take advantage of the woven mesh structure and the exponential dependence of resistance change on the GWF's geometry. This strain sensor with good repeatability and high sensitivity may provide an economical sensing modality for microcontrol applications. To extend the domain of application of the current GWF strain sensors, future work will focus on the correlation between the electromechanical properties and the polycrystalline feature (e.g. crystallinity, shape and size of graphene domains) of GWFs.

\section{Methods}

Synthesis of GWFs. GWFs were prepared from atmospheric pressure CVD by using a woven copper mesh as the template and methane as the carbon source ${ }^{22}$. After etching away copper in an aqueous solution of $\mathrm{FeCl}_{3}(0.5 \mathrm{~mol} / \mathrm{L})$ and $\mathrm{HCl}$ $(0.5 \mathrm{~mol} / \mathrm{L})$, the freestanding GWF film was transferred to deionized water and rinsed thoroughly for later use.
Characterizations. GWF-on-PDMS structures for strain sensor characterization were fabricated on a rectangular-shaped backing structure made of PDMS. After setting and drying the GWF film on PDMS, lead wires were connected it using silver paste. Tensile characterizations of the sensors were performed on a computer controlled, home-made actuating unit located on an optical bench. Surface structure evaluations of the GWFs (cracks, wrinkles) were performed using an optical microscope (Olympus BX51M) and a scanning electron microscope (S-4800, Hitachi).

Fracture model and resistance predication of GWF upon stretching. If the GWF consists of $m$ horizontal GMRs and $n$ vertical GMRs, it can be modeled as a $2 \mathrm{D}$ resistor network as shown in Figure $4 \mathrm{~b}$. To measure the resistance from the left side of GWF to its right side, the nodes on the edges of both sides are shorted, respectively. To investigate the effect of the fracture of GMRs on the GWF's resistance, a program was developed to obtain the relationship between the resistance of GWF and the tensile strain. The program has as input a data file that includes the position and the crack information in each cracked GMR. The program employs the technique of nodal analysis to form the linear equation system ${ }^{26}$. With other sparse matrix functions of MATLAB, this program is able to efficiently handle large-scale GWF and provide an easy-to-use tool to estimate the GWF's resistance for specified cracked GMRs. To investigate the effect of the crack density on the GWF's resistance, the function of the program is enhanced to output the resistance for a specified crack density. This is accomplished by randomly generating the positions and crack informations of the cracked GMRs.

1. Herrmann, J., Müller, K.-H., Reda, T., Baxter, G. R., Raguse, B., de Groot, G. J. J. B., Chai, R., Roberts, M. \& Wieczorek, L. Nanoparticle films as sensitive strain gauges. Appl. Phys. Lett. 91, 183105 (2007).

2. Obitayo, W. \& Liu, T. A Review: Carbon nanotube-based piezoresistive strain sensors. J. Sensors 2012, 652438 (2012).

3. Fernández-Regúlez, M., Plaza, J. A., Lora-Tamayo, E. \& Paulo, A. S. Lithography guided horizontal growth of silicon nanowires for the fabrication of ultrasensitive piezoresistive strain gauges. Microelectr. Engin. 87, 1270 (2010).

4. Zhou, J., Gu, Y. D., Fei, P., Mai, W. J., Gao, Y. F., Yang, R. S., Bao, G. \& Wang, Z. L. Flexible piezotronic strain sensor. Nano Lett. 8, 3035 (2008).

5. Xiao, X., Yuan, L. Y., Zhong, J. W., Ding, T. P., Liu, Y., Cai, Z. X., Rong, Y. G., Han, H. W., Zhou, Y. \& Wang, Z. L. High-strain sensors based on ZnO nanowire/ polystyrene hybridized flexible films. Adv. Mater. 23, 5440 (2011).

6. Loh, K. J., Kim, J., Lynch, J. P., Kam, N. W. S. \& Kotov, N. A. Multifunctional layerby-layer carbon nanotube-polyelectrolyte thin films for strain and corrosion sensing. Smart Mater. Structures 16, 429 (2007).

7. Song, X. H., Liu, S., Gan, Z. Y., Lv, Q., Cao, H. \& Yan, H. Controllable fabrication of carbon nanotube-polymer hybrid thin film for strain sensing. Microelectronic Engin. 86, 2330 (2009).

8. Lipomi, D. J., Vosgueritchian, M., Tee, B. C. K., Hellstrom, S. L., Lee, J. A., Fox, C. H. $\&$ Bao, Z. N. Skin-like pressure and strain sensors based on transparent elastic films of carbon nanotubes. Nature Nanotechnol. 6, 788 (2011).

9. Zhang, Y. Y., Sheehan, C. J., Zhai, J. Y., Zou, G. F., Luo, H. M., Xiong, J., Zhu, Y. T. \& Jia, Q. X. Polymer-embedded carbon nanotube ribbons for stretchable conductors. Adv. Mater. 22, 3027 (2010). 
10. hao, H. B., Zhang, Y. Y., Bradford, P. D., Zhou, Q. A., Jia, Q. X., Yuan, F. G. \& Zhu, Y. T. Carbon nanotube yarn strain sensors. Nanotechnol. 21, 305502 (2010).

11. Zhang, J., Liu, J. W., Zhuang, R. C., Mader, E., Heinrich, G. \& Gao, S. L. Single MWNT-glass fiber as strain sensor and switch. Adv. Mater. 23, 3392 (2011).

12. Yamada, T., Hayamizu, Y., Yamamoto, Y., Yomogida, Y., Izadi-Najafabadi, A., Futaba, D. N. \& Hata, K. A stretchable carbon nanotube strain sensor for humanmotion detection. Nature Nanotechnol. 6, 296 (2011).

13. Yin, G., Hu, N., Karube, Y., Liu, Y. L., Li, Y. \& Fukunaga, H. A carbon nanotube/ polymer strain sensor with linear and anti-symmetric piezoresistivity. J. Composite Mater. 45, 1315 (2011).

14. Rein, M. D., Breuer, O. \& Wagner, H. D. Sensors and sensitivity: Carbon nanotube buckypaper films as strain sensing devices. Composites Sci. Technol. 71, 373 (2011).

15. Sakhaee-Pour, A., Ahmadian, M. T. \& Vafai, A. Potential application of singlelayered graphene sheet as strain sensor. Solid State Commun. 147, 336 (2008).

16. Kumar, S. B. \& Guo, J. Strain-induced conductance modulation in graphene grain boundary. Nano Lett. 12, 1362 (2012).

17. Klimov, N. N., Jung, S., Zhu, S. Z., Li, T., Wright, C. A., Solares, S. D., Newell, D. B., Zhitenev, N. B. \& Stroscio, J. A. Electromechanical properties of graphene drumheads. Science 336, 1557 (2012).

18. Wang, Y., Yang, R., Shi, Z. W., Zhang, L. C., Shi, D. X., Wang, E. \& Zhang, G. Y. Super-elastic graphene ripples for flexible strain sensors. ACS Nano 5, 3645 (2011).

19. Xie, X. J., Bai, H., Shi, G. Q. \& Qu, L. T. Load-tolerant, highly strain-responsive graphene sheets. J. Mater. Chem. 21, 2057 (2011).

20. Chen, X., Zheng, X. H., Kim, J. K., Li, X. X. \& Lee, D. W. Investigation of graphene piezoresistors for use as strain gauge sensors. J. Vac. Sci. Technol. B 29, 06 FE01 (2011).

21. Eswaraiah, V., Balasubramaniam, K. \& Ramaprabhu, S. Functionalized graphene reinforced thermoplastic nanocomposites as strain sensors in structural health monitoring. J. Mater. Chem. 21, 12626 (2011).

22. Li, X., Sun, P. Z., Fan, L. L., Zhu, M., Wang, K. L., Zhong, M. L., Wei, J. Q., Wu, D. H., Cheng, Y. \& Zhu, H. W. Multifunctional graphene woven fabrics. Scientific Reports 2, 395 (2012).
23. Dobie, W. B. \& Isaac Peter, C. G. Electric Resistance Strain Gauges (English Universities Press Limited, 1948).

24. Mattmann, C., Clemens, F. \& Tröster, G. Sensor for measuring strain in textile. Sensors 8, 3719 (2008).

25. Cao, J., Wang, Q. \& Dai, H. J. Electromechanical properties of metallic, quasimetallic, and semiconducting carbon nanotubes under stretching. Phys. Rev. Lett. 90, 157601 (2003).

26. Pillage, L. T., Rohrer, R. A. \& Visweswariah, C. Electronic circuit and system simulation methods. McGRAW-HILL Book Co. (1995).

\section{Acknowledgements}

This work was supported by the National Science Foundation of China (50972067) and the Beijing Natural Science Foundation (2122027). We thank Dr. J. R. Yang for helpful discussions.

\section{Author contributions}

H.W.Z. and X.L. conceived and designed the experiments. X.L., R.J.Z. performed the experiments. W.J.Y. and Q.S.Z. conducted the theoretical analysis. All authors interpreted the results. H.W.Z., Q.S.Z. and R.S.R. co-wrote the manuscript.

\section{Additional information}

Supplementary information accompanies this paper at http://www.nature.com/ scientificreports

Competing financial interests: The authors declare no competing financial interests.

License: This work is licensed under a Creative Commons

Attribution-NonCommercial-NoDerivs 3.0 Unported License. To view a copy of this license, visit http://creativecommons.org/licenses/by-nc-nd/3.0/

How to cite this article: $\mathrm{Li}$, X. et al. Stretchable and highly sensitive graphene-on-polymer strain sensors. Sci. Rep. 2, 870; DOI:10.1038/srep00870 (2012). 